\title{
Tratamiento radical mínimamente invasivo en cáncer de cuello uterino invasor: experiencia de la Clínica Ginecotocológica A
}

\author{
Sebastián Ben*, Diego Greif*, María Fernanda Nozar*, Juan Calderón†, \\ María Ximena Fernández†
}

\section{Resumen}

Introducción: el cáncer de cuello uterino es el cuarto más frecuente a nivel mundial en mujeres. En estadios precoces la cirugía es el tratamiento de elección. El tratamiento quirúrgico estándar es la histerectomía radical tipo C1 y linfadenectomía pélvica. La vía de abordaje clásicamente utilizada en Uruguay es la laparotomía. Los resultados oncológicos por las diferentes vías de abordaje se consideran equiparables. Presentamos la primera serie de casos publicada en Uruguay de tratamiento quirúrgico de mínima invasión en cáncer de cuello uterino.

Método: estudio descriptivo prospectivo de serie de casos. Se incluyen todas las pacientes operadas por cáncer de cuello uterino invasor como tratamiento primario con vía de abordaje de mínima invasión, desde enero de 2013 hasta diciembre de 2016 en la Clínica Ginecotocológica A del Centro Hospitalario Pereira Rossell.

Resultados: se operaron 17 pacientes: 14 histerectomía radical laparoscópica + linfadenectomía pélvica laparoscópica y 3 histerectomía radical vaginal + linfadenectomía pélvica laparoscópica. Edad media: 44 años. Tiempo quirúrgico medio: 261 minutos. Tiempo medio de internación posoperatoria: cinco días. Conversión a laparotomía: dos casos. Se resecaron una media de 14,4 ganglios pélvicos. Ningún caso con márgenes comprometidos. Hubo dos lesiones vesicales. Ningún caso de sangrado severo, transfusión, lesión intestinal, vascular ni ureteral. No hubo ningún caso de infección, dehiscencia, trombosis, reintervención o readmisión. No hubo recurrencias, con media de seguimiento de 29 meses.

Conclusiones: la cirugía de mínima invasión es factible para el tratamiento del cáncer de cuello uterino en estadios precoces en nuestro medio, obteniendo un buen resultado anatomopatológico, quirúrgico y oncológico con baja morbilidad.

Palabras clave: NEOPLASIAS DEL CUELLO UTERINO

PROCEDIMIENTOS QUIRÚRGICOS MÍNIMAMENTE INVASIVOS

HISTERECTOMÍA

ESCISIÓN DEL GANGLIO LINFÁTICO

Key words: UTERINE CERVICAL NEOPLASMS

MINIMALLY INVASIVE SURGICAL PROCEDURES

HYSTERECTOMY

LYMPH NODE EXCISION

\footnotetext{
* Profesor Adjunto Clínica Ginecotocológica A.

† Ex Residente Clínica Ginecotocológica A.

Clínica Ginecotocológica A. Prof. Dr. Leonel Briozzo. Facultad de Medicina. Universidad de la República Oriental del Uruguay.

Correspondencia: Sebastián Ben. Bulevar Artigas 1550. Montevideo, Uruguay. Correo electrónico:ben.sebastian@hotmail.com

No hubo fuentes de apoyo económico.

Los autores declaran no tener conflicto de interés.

Recibido: $2 / 1 / 18$

Aprobado 2/4/18
} 


\section{Introducción}

El cáncer de cuello uterino es el cuarto más frecuente a nivel mundial en mujeres, con una incidencia estimada de 528.000 casos por año, siendo el $85 \%$ de los casos en países con bajo desarrollo ${ }^{(1)}$. En Uruguay la incidencia es de 15,28 casos/100.000 de tasa estandarizada por edad con 327 casos nuevos por año. La mortalidad es de 5,33 casos/100.000 de tasa estandarizada por edad con 134 muertes anuales ${ }^{(2)}$.

Existen diversas opciones terapéuticas en función del estadio clínico, factores pronósticos y los deseos reproductivos de la paciente, e incluyen: cirugía (conización, traquelectomía simple o radical, histerectomía simple extrafascial o radical), radioterapia (RDT) y quimioterapia (QMT).

En estadios precoces (estadios FIGO IA, IB1 y IIA1) la cirugía y la RDT ofrecen resultados similares. Sin embargo, se considera de elección el tratamiento quirúrgico, aunque la RDT es una alternativa válida en función de la experiencia del centro y de las condiciones de la paciente. El tratamiento quirúrgico ofrece ventajas como la conservación de la función ovárica, mantiene una vagina más funcional y facilita el conocimiento de los factores pronósticos anatomopatológicos ${ }^{(3)}$.

En el estadio FIGO IA1 (microinvasor) el tratamiento estándar es la histerectomía radical tipo A según la clasificación de Querleu y Morrow (histerectomía simple extrafascial). Para los estadios IA2, IB1 y IIA1 el tratamiento quirúrgico estándar en las pacientes sin deseo de fertilidad es la histerectomía radical tipo $\mathrm{C} 1$ (histerectomía radical, colpectomía de tercio superior, parametrectomía con preservación de nervios autonómicos pélvicos) y linfadenectomía pélvica ${ }^{(4,5)}$.

En estadios localmente avanzados (IB2, IIA2-IV) el tratamiento de elección es la asociación RDT-QMT con finalidad radical, ampliada en ocasiones a las áreas ganglionares lumboaórticas ${ }^{(6)}$.

La vía de abordaje clásicamente utilizada en Uruguay es la laparotomía. Los resultados oncológicos ofrecidos por las diferentes vías de abordaje (vaginal, abdominal, laparoscópica) se consideran equiparables si son practicadas por personal con suficiente experiencia ${ }^{(7)}$. Las vías de abordaje de mínima invasión para el tratamiento del cáncer de cuello uterino en estadios precoces es segura y no tiene diferencias con la vía laparotómica en cuanto a recurrencia y sobrevida ${ }^{(8-19)}$. Se entiende por cirugía de mínima invasión el conjunto de técnicas terapéuticas que mediante visión directa, o endoscópica, o por otras técnicas de imagen, utiliza vías naturales o mínimos abordajes para introducir herramientas y actuar en distintos territorios de la economía humana. La cirugía de mínima invasión se asocia a menor pérdida hemá- tica, menor necesidad de transfusión, menor requerimiento analgésico, estadía hospitalaria más corta, mejor resultado estético, retorno precoz laboral y a las actividades diarias ${ }^{(5-7)}$.

Presentamos la primera serie de casos publicada en Uruguay de tratamiento quirúrgico de mínima invasión de cáncer de cuello uterino.

\section{Material y método}

Estudio descriptivo prospectivo de serie de casos en la cual se incluyen todas las pacientes operadas por cáncer de cuello uterino invasor como tratamiento primario con una vía de abordaje de mínima invasión, desde enero de 2013 hasta diciembre de 2016 en la Clínica Ginecotocológica A en el Hospital de la Mujer del Centro Hospitalario Pereira Rossell, institució de referencia nacional en ginecología oncológica.

El equipo quirúrgico estuvo integrado en todos los casos por los mismos tres miembros del equipo de la Clínica Ginecotocológica A. Los tres miembros son profesores adjuntos titulares de la Clínica Ginecotocológica A, encargados del área de oncología con formación específica nacional e internacional en cirugía de mínima invasión y ginecología oncológica.

En todos los casos fue obtenido el consentimiento informado luego de haber sido explicadas las diferentes opciones terapéuticas, los riesgos del procedimiento quirúrgico y la posibilidad de conversión a laparotomía.

Todas las pacientes tenían confirmación anatomopatológica de carcinoma invasor mediante biopsia o conización. Se realizó la anamnesis completa, examen físico que incluye tacto rectovaginal por dos integrantes del equipo e imagenología mediante resonancia magnética (RM).

Los criterios de inclusión fueron aquellas pacientes sin contraindicaciones médicas para cirugía, tumores menores a $4 \mathrm{~cm}$ de diámetro confinados al cuello del útero, sin compromiso parametrial y sin sospecha de compromiso ganglionar o a distancia en la RM, o ambos.

El tipo de vía de abordaje de mínima invasión utilizado fue la laparoscopía (histerectomía radical tipo $\mathrm{C} 1$ y linfadenectomía pélvica laparoscópica) y la vía vaginal asociada a la laparoscopía (operación de Schauta).

La histerectomía radical laparoscópica se realizó con anestesia general y con la paciente en posición de litotomía. En primer término se efectuó la linfadenectomía pélvica bilateral y posteriormente la histerectomía radical.

La operación de Schauta incluye en primer lugar la linfadenectomía pélvica bilateral laparoscópica y posteriormente la histerectomía radical por vía vaginal.

El abordaje laparoscópico se realizó con técnica cerrada mediante aguja de Veress a nivel umbilical, y cua- 


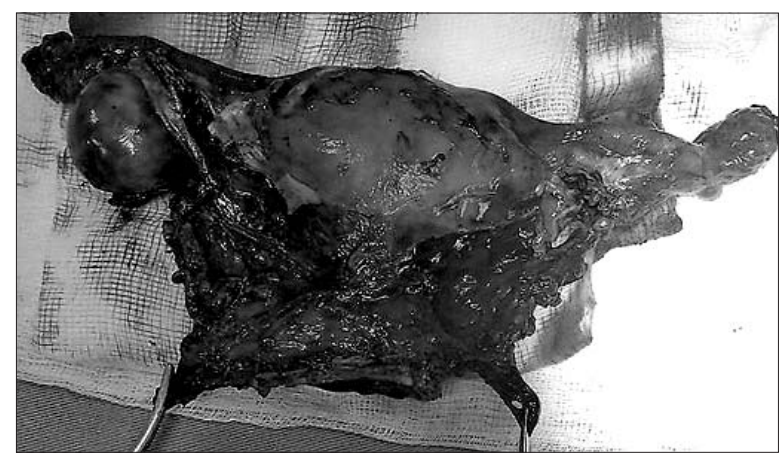

Figura 1. Pieza de histerectomía radical laparoscópica

tro trocares accesorios a nivel de fosas ilíacas y flancos. Se utilizó en todas las cirugías energía bipolar con Ligasure ${ }^{\circ}$ y pinza de agarre bipolar ROBI ${ }^{\circledR}$ de Kelly Clermont-Ferrand.

Se realizó salpingectomía bilateral a todas las pacientes. El manejo de los ovarios dependió de la edad, tipo histológico, factores de riesgo de cáncer de ovario y de la preferencia de las pacientes.

La función urinaria en el posoperatorio alejado fue valorada clínicamente mediante el interrogatorio (incontinencia, dificultad para evacuación, urgencia miccional, necesidad de cateterismo, disuria, retención aguda de orina).

\section{Resultados}

Desde enero de 2013 hasta diciembre de 2016 se operaron 17 pacientes con diagnóstico de cáncer de cuello uterino invasor mediante cirugía de mínima invasión, 14 histerectomía radical laparoscópica tipo C1 + linfadenectomía pélvica laparoscópica y tres con histerectomía radical vaginal (operación de Schauta) + linfadenectomía pélvica laparoscópica (figura 1).

La edad media fue de 44 años (24-69). La procedencia fue siete pacientes de Montevideo y diez del interior del país. Seis de ellas referían tabaquismo actual, diez negaban tabaquismo y una extabaquista. En cuanto a la paridad, 12 casos fueron multíparas, cuatro con 1-2 gestaciones previas y un caso nulípara. En cuanto al motivo de consulta: hallazgo de screening diez casos, genitorragia cuatro casos, sinusorragia un caso, dolor un caso y tumoración un caso. La histología que presentaron fue escamosa en todas las pacientes. Los estadios al momento de la cirugía fueron: IA2 cinco casos y IB1 doce casos (tabla 1).

El tiempo quirúrgico medio fue de 261 minutos (190-410). El tiempo medio de internación posoperatoria fue de 5 días (4-7). Se convirtieron a la vía laparotómica dos casos, uno por mal funcionamiento del equipamiento y otro por mala tolerancia de la paciente al neumoperitoneo.
Tabla 1. Características de la población

\begin{tabular}{|c|c|}
\hline Pacientes & $n=17$ \\
\hline Edad (años) & Media 44 (24-69) \\
\hline \multicolumn{2}{|l|}{ Procedencia } \\
\hline Montevideo & 7 \\
\hline Interior & 10 \\
\hline \multicolumn{2}{|l|}{ Tabaquismo } \\
\hline Sí & 6 \\
\hline No & 10 \\
\hline Extabaquista & 1 \\
\hline \multicolumn{2}{|l|}{ Paridad } \\
\hline Nuligesta & 1 \\
\hline 1-2 gestas & 4 \\
\hline 3 o más gestas & 12 \\
\hline \multicolumn{2}{|l|}{ Motivo de consulta } \\
\hline Screening & 10 \\
\hline Genitorragia & 4 \\
\hline Sinusorragia & 1 \\
\hline Tumoración & 1 \\
\hline Dolor & 1 \\
\hline \multicolumn{2}{|l|}{ Estadio } \\
\hline IA2 & 5 \\
\hline IB1 & 12 \\
\hline \multicolumn{2}{|l|}{ Histología } \\
\hline Escamosos & 17 \\
\hline
\end{tabular}

Se realizó preservación nerviosa en todos los casos mediante disección del plexo hipogástrico y conservación de ramas nerviosas en el parametrio posterior, preservación del plexo esplácnico a nivel de parametrio lateral mediante disección parametrial hasta la vena uterina profunda y preservación de ramas de plexo hipogástrico a nivel de parametrio ventral por debajo de uréter en su trayecto anterior.

La linfadenectomía pélvica se realizó de forma completa y sistematizada de ganglios ilíacos comunes, ilíacos externos, ilíacos internos, interilíacos y obturatrices. Se resecaron una media de 14,4 ganglios pélvicos. Se identificó bilateralmente el nervio obturatriz indemne en todos los casos. En uno de los 17 casos se encontró metástasis linfática. 
Tabla 2. Resultados quirúrgicos de las pacientes operadas por cáncer de cuello uterino invasor con cirugía de mínima invasión

\begin{tabular}{lc}
\hline Tiempo quirúrgico medio (minutos) & $261(190-410)$ \\
Días de internación posoperatorio (media) & $5(4-7)$ \\
Conversión a laparotomía ( $\mathrm{n})$ & $2 / 17$ \\
Ganglios resecados (media) & 14,4 \\
Compromiso de márgenes ( $\mathrm{n})$ & 0 \\
Lesión vesical ( $\mathrm{n}$ ) & $2 / 17$ \\
Sangrado severo/transfusiones & 0 \\
Otras complicaciones mayores & 0 \\
\hline
\end{tabular}

En ningún caso hubo compromiso de márgenes en la pieza anatomopatológica. No hubo compromiso parametrial ni vaginal en el estudio de la patología. Se realizó conservación de ovarios en las pacientes menores de 45 años. No se encontraron metástasis en trompas de Falopio ni en los ovarios resecados.

En cuanto a las complicaciones intraoperatorias hubo dos lesiones vesicales al momento de la disección del espacio vesico-vaginal que se resolvieron por vía laparoscópica con sutura de poliglactina 910 3-0 puntos separados extamucosos en dos planos sin complicaciones posoperatorias (tabla 2). Ambas pacientes con antecedente de cesáreas, conización y traquelectomía previa a cirugía. En ningún caso hubo sangrado intraoperatorio severo, requerimiento de transfusión, lesión intestinal, vascular, ni lesión ureteral. Para reducir el riesgo de lesión ureteral se realizó cateterización con catéter doble J en los primeros diez casos. Se realizó una cuidadosa disección de uréteres utilizando la menor cantidad de energía posible. No hubo ningún caso de infección, dehiscencia, trombosis ni necesidad de reintervención, ni readmisión hospitalaria. No hubo complicaciones miccionales crónicas. En cuanto al tiempo libre de recurrencia y sobrevida global no hubo recurrencia en las pacientes operadas con una media de seguimiento de 29 meses. No hubo pérdida de seguimiento en las $17 \mathrm{pa}-$ cientes.

\section{Discusión}

A pesar de los métodos de detección precoz y de las estrategias para disminución de su incidencia, el cáncer de cuello uterino continúa siendo un problema de salud en Uruguay y en el mundo. El tratamiento para los estadios precoces es de elección quirúrgico. Con el objetivo de disminuir la morbilidad quirúrgica, sin comprometer el resultado oncológico, surgen las técnicas de mínima invasión que han demostrado seguridad en dichos aspectos. La histerectomía radical laparoscópica en cáncer de cuello uterino fue inicialmente descrita por Canis y colaboradores en $1990^{(10,11)}$. El desarrollo del instrumental quirúrgico y la cadena de visión, así como el aprendizaje y entrenamiento en laparoscopía del equipo quirúrgico actuante, permite realizar este procedimiento de alta complejidad en nuestro medio. Presentamos la primera serie de casos publicada en Uruguay de tratamiento de mínima invasión en cáncer de cuello uterino invasor.

En nuestra serie la histerectomía radical tipo $\mathrm{C} 1$ se realizó para los estadios invasores precoces que incluye la histerectomía radical con preservación de nervios autonómicos pélvicos importantes para la función miccional, rectal y sexual. La preservación nerviosa demostró tener seguridad oncológica ${ }^{(20,21)}$.

El tiempo quirúrgico promedio fue de 261 minutos. Una revisión de Geetha y colaboradores que incluyó 21 estudios de histerectomía radical laparoscópica mostró un tiempo quirúrgico medio de 259 minutos, similar al de nuestra serie ${ }^{(22)}$. Algunos autores, como Putambekar, publican resultados con tiempo medio de cirugía de 92 minutos, si bien estos resultados de tiempo no están reportados en otras series ${ }^{(23)}$. Este procedimiento es considerado una cirugía de alta complejidad, por lo que se requiere una curva de aprendizaje específica. Se propone para esta técnica alrededor de $18-20 \operatorname{casos}^{(24)}$. En nuestra serie mostramos los primeros 17 casos realizados, por lo que forma parte de nuestra curva de aprendizaje.

El tiempo medio de internación fue de cinco días. Este tipo de cirugía tiene beneficios en el tiempo de internación, algunos autores plantean incluso la posibilidad que sea una cirugía ambulatoria ${ }^{(25)}$. En nuestro caso hay que destacar que un porcentaje importante de las pacientes operadas eran del interior y por ende requieren controles más cercanos, lo que dificulta el alta precoz. En la revisión de Geetha y colaboradores la media de internación fue de 5,5 días, similar a la de nuestra serie ${ }^{(22)}$.

En ningún caso hubo compromiso de márgenes, se ha descrito una media de compromiso de márgenes de $3,5 \%$. En la linfadenectomía pélvica se obtuvo una media de 14,4 ganglios. Internacionalmente se ha reportado una media fue de 21 ganglios pélvicos resecados ${ }^{(22)}$. Es de destacar en este punto que lo relevante es la representatividad de las cadenas ganglionares, lo cual se obtuvo en nuestra serie de casos. Por otro lado, el número de ganglios reportados depende del procedimiento quirúrgico, del biotipo de la paciente y de la técnica anatomopatológica utilizada en su contabilización.

De las 17 pacientes en dos casos hubo conversión a laparotomía (12\%), vinculado a mala tolerancia a neumoperitoneo y a problema eléctrico del instrumental. Se 
ha reportado una tasa de conversión de $1,5 \%$ en la serie de Park y colaboradores ${ }^{(26)}$. Destacamos que las conversiones a laparotomía no fueron vinculadas a dificultades de la técnica específica sino a situaciones vinculadas al paciente o al instrumental.

Con respecto al sangrado severo y la necesidad de transfusiones está descrita una media de 0,8\%-2,7\%, en nuestra serie ninguna paciente requirió transfusión de glóbulos rojos $^{(22,27)}$.

No hubo complicaciones urinarias crónicas en nuestra serie. La frecuencia es variable durante la histerectomía radical de acuerdo con las diferentes series (8\%-80\%). Las complicaciones urinarias se presentan significativamente disminuidas al realizar la preservación nerviosa. No contamos con evaluación de la función sexual o defecatoria en nuestro estudio. Las mismas se pueden ver minimizadas al realizar la técnica de preservación nerviosa ${ }^{(28)}$.

En nuestra serie hubo dos lesiones vesicales que se resolvieron sin inconvenientes por vía laparoscópica con sutura laparoscópica. Destacamos que estos dos casos presentaban antecedentes quirúrgicos que aumentan el riesgo de lesiones vesicales: cesárea, conización y traquelectomía. No hubo complicaciones infecciosas posoperatorias. El riesgo de infección descrito en el posoperatorio fue de $6,5 \%{ }^{(22,29)}$.

No hubo ningún caso de lesión ureteral. Para disminuir el riesgo se colocaron catéteres doble J ureterales previo a la cirugía durante los primeros diez casos; si bien este procedimiento puede dar más seguridad para la disección ureteral, provoca cierta "rigidez" de los mismos que dificulta la disección, por lo que no se utilizó en los casos posteriores.

No hubo ningún caso de recurrencia con un seguimiento medio de 29 meses. La tasa de recurrencia media para cáncer de cuello en estadios precoces es de 5\%, siendo esta similar en la vía tradicional laparotómica y en la vía de mínima invasión ${ }^{(22,28)}$. En nuestra serie contamos con un seguimiento medio mayor a dos años, siendo este el momento de mayor riesgo de recurrencia.

\section{Conclusiones}

La cirugía de mínima invasión es factible para el tratamiento del cáncer de cuello uterino en estadios precoces en nuestro medio, obteniendo un buen resultado anatomopatológico, quirúrgico y oncológico con baja morbilidad.

\section{Abstract}

Introduction: Cervical cancer is the fourth most frequent cancer in women around the world. Surgery is the first choice for treatment in early stages.
The standard surgical treatment is Type C1 Radical Hysterectomy and pelvic lymphadenectomy. Laparotomy is the typical surgical approach used in Uruguay. Oncologic results are similar for the different surgical approaches. The study presents the first case series of minimally invasive cervical treatment of cervical cancer published in Uruguay.

Method: descriptive, prospective study of a series of cases. All patients operated at the Gynecological Clinic "A", Pereira Rossell Hospital from January 2013 until December 2016 for invasive cervical cancer with minimally invasive approach as the primary treatment were included in the study.

Results: 17 patients were operated: 14 Radical Laparoscopic hysterectomy + laparoscopic pelvic lymphadenectomy and 3 radical vaginal hysterectomy + laparoscopic pelvic lymphadenectomy. Average ager: 44 years old. Average surgical time: 261 minutes. Average post-surgery hospital stay: 5 days. 2 cases changed into laparotomy. An average of 14.4 pelvic lymph nodes were resected. In no case were margins compromised. Two bladder lesions, no cases of severe bleeding, transfusion, intestine, vascular or ureteral lesions. No cases of infection, deshicence, thrombosis, reoperation or readmission. NO recurrence, average follow up was 29 months.

Conclusions: minimally invasive surgery is a feasible treatment for early stages cervical cancer in our country, obtaining good anatomopathological, surgical and oncological results, morbility being low.

\section{Resumo}

Introdução: O câncer de colo uterino é o quarto tipo de câncer mais frequente entre mulheres em todo o mundo. Em estádios precoces a cirurgia é o tratamento de eleição. O tratamento cirúrgico padrão é a Histerectomia Radical Tipo $\mathrm{C} 1$ e a linfadenectomia pélvica. No Uruguai a via de abordagem classicamente utilizada é a laparotomia. Os resultados oncológicos pelas diferentes vias de abordagem são considerados equivalentes. Apresentamos a primeira serie de casos publicados no Uruguai, de tratamento cirúrgico minimamente invasivo no câncer de colo uterino.

Métodos: Estudo descritivo prospectivo de serie de casos. Foram incluídas todas as pacientes operadas por câncer de colo uterino invasor como tratamento primário com via de abordagem minimamente invasiva, no período janeiro de 2013 - dezembro de 2016 na Clínica Ginecotocológica "A”, Hospital Pereira Rossell.

Resultados: foram operadas 17 pacientes: 14 Histerectomia Radical Laparoscópica + linfadenectomia pélvica laparoscópica e 3 Histerectomia Radical Vaginal + linfadenectomia pélvica laparoscópica. Idade media: 44 
anos. Tempo médio da cirurgia: 261 minutos. Tempo médio de internação pós-operatória: 5 dias. Conversão a laparotomia: 2 casos. Foi ressecada uma média de 14,4 gânglios pélvicos. Nenhum caso apresentou comprometimento das margens. Foram registradas 2 lesões vesicais. Não se observaram casos de sangramento severo, transfusão, lesão intestinal, vascular nem ureteral. Não se registraram casos de infecção, deiscência, tromboses, intervenção ou reinternação. Não foram registradas recorrências, com um seguimento médio de 29 meses.

Conclusões: a cirurgia minimamente invasiva é factível para o tratamento do câncer de colo uterino em estádios precoces no nosso meio, mostrando bons resultados anatomopatológicos, cirúrgicos e oncológicos com baixa morbidade.

\section{Bibliografía}

1. Ferlay J, Soerjomataram I, Ervik M, Dikshit R, Eser S, Mathers C, et al. GLOBOCAN 2012: estimated cancer incidence, mortality and prevalence worldwide in 2012. Disponible en: http://globocan.iarc.fr [Consulta: $1^{\circ}$ dic. 2017].

2. Barrios E, Garau M, Alonso R, Musetti C. IV Atlas de incidencia de cáncer en el Uruguay 2007-2011. Montevideo: CHLCC, 2014.

3. Landoni F, Maneo A, Colombo A, Placa F, Milani R, Perego $\mathbf{P}$, et al. Randomised study of radical surgery versus radiotherapy for stage Ib-IIa cervical cancer. Lancet 1997; 350(9077):535-40.

4. National Comprehensive Cancer Network. Cancer cervix. 2018. Disponible en: https://www.nccn.org/professionals/physician_gls/pdf/cervical.pdf. [Consulta: $1^{\circ}$ dic. 2017]

5. Querleu D, Morrow CP. Classification of radical hysterectomy. Lancet Oncol 2008; 9(3):297-303.

6. Pearcey R, Brundage M, Drouin P, Jeffrey J, Johnston D, Lukka H, et al. Phase III trial comparing radical radiotherapy with and without cisplatin chemotherapy in patients with advanced squamous cell cancer of the cervix. J Clin Oncol 2002; 20:966-72.

7. Abu-Rustum NR, Gemignani ML, Moore K, Sonoda Y, Venkatraman E, Brown C, et al. Total laparoscopic radical hysterectomy with pelvic lymphadenectomy using the argon-beam coagulator: pilot data and comparison to laparotomy. Gynecol Oncol 2003; 91:402-9.

8. Magrina JF. Outcomes of laparoscopic treatment for endometrial cancer. Curr Opin Obstet Gynecol 2005; 17:343-6.

9. Magrina JF, Mutone NF, Weaver AL, Magtibay PM, Fowler RS, Cornella JL. Laparoscopic lymphadenectomy and vaginal or laparoscopic hysterectomy with bilateral salpingo-oophorectomy for endometrial cancer: morbidity and survival. Am J Obstet Gynecol 1999; 181:376-81.

10. Canis M, Mage G, Wattiez A, Pouly JL, Manhes H, Bruhat MA. Does endoscopic surgery have a role in radical surgery of cancer of the cervix uteri? J Gynecol Obstet Biol Reprod (Paris) 1990; 19(7):921.
11. Nezhat CR, Burrell MO, Nezhat FR, Benigno BB, Welander CE. Laparoscopic radical hysterectomy with paraaortic and pelvic node dissection. Am J Obstet Gynecol 1992; 166(3):864-5.

12. Canis M, Mage G, Pouly JL, Pomel C, Wattiez A, Glowaczover $\mathbf{E}$, et al. Laparoscopic radical hysterectomy for cervical cancer. Baillieres Clin Obstet Gynaecol 1995; 9(4):675-89.

13. Spirtos NM, Schlaerth JB, Kimball RE, Leiphart VM, Ballon SC. Laparoscopic radical hysterectomy (type III) with aortic and pelvic lymphadenectomy. Am J Obstet Gynecol 1996; 174(6):1763-7.

14. Spirtos NM, Eisenkop SM, Schlaerth JB, Ballon SC. Laparoscopic radical hysterectomy (type III) with aortic and pelvic lymphadenectomy in patients with stage I cervical cancer: surgical morbidity and intermediate follow-up. Am J Obstet Gynecol 2002; 187:340-8.

15. Abu-Rustum NR, Gemignani ML, Moore K, Sonoda Y, Venkatraman E, Brown C, et al. Total laparoscopic radical hysterectomy with pelvic lymphadenectomy using the argon-beam coagulator: pilot data and comparison to laparotomy. Gynecol Oncol 2003; 91(2):402-9.

16. Ramirez PT, Slomovitz BM, Soliman PT, Coleman RL, Levenback C. Total laparoscopic radical hysterectomy and lymphadenectomy: the M. D. Anderson Cancer Center experience. Gynecol Oncol 2006; 102(2):252-5.

17. Gil-Moreno A, Díaz-Feijoo B, Roca I, Puig O, Pérez-Benavente MA, Aguilar I, et al. Total laparoscopic radical hysterectomy with intraoperative sentinel node identification in patients with early invasive cervical cancer. Gynecol Oncol 2005; 96(1):187-93.

18. Frumovitz M, dos Reis R, Sun CC, Milam MR, Bevers MW, Brown J, et al. Comparison of total laparoscopic and abdominal radical hysterectomy for patients with early-stage cervical cancer. Obstet Gynecol 2007; 110(1):96-102.

19. Hong JH, Choi JS, Lee JH, Eom JM, Ko JH, Bae JW, et al. Can laparoscopic radical hysterectomy be a standard surgical modality in stage IA2-IIA cervical cancer? Gynecol Oncol 2012; 127(1):102-6.

20. van Gent MD, Romijn LM, van Santen KE, Trimbos JB, de Kroon CD. Nerve-sparing radical hysterectomy versus conventional radical hysterectomy in early-stage cervical cancer. A systematic review and meta-analysis of survival and quality of life. Maturitas 2016; 94:30-8.

21. Mendivil AA, Rettenmaier MA, Abaid LN, Brown JV 3rd, Micha JP, Lopez KL, et al. Survival rate comparisons amongst cervical cancer patients treated with an open, robotic-assisted or laparoscopic radical hysterectomy: a five year experience. Surg Oncol 2016; 25(1):66-71.

22. Geetha P, Nair MK. Laparoscopic, robotic and open method of radical hysterectomy for cervical cancer: a systematic review. J Minim Access Surg 2012; 8(3):67-73.

23. Puntambekar SP, Palep RJ, Puntambekar SS, Wagh GN, Patil AM, Rayate NV, et al. Laparoscopic total radical 
hysterectomy by the Pune technique: our experience of 248 cases. J Minim Invasive Gynecol 2007; 14(6):682-9.

24. Kong TW, Chang SJ, Paek J, Park H, Kang SW, Ryu HS. Learning curve analysis of laparoscopic radical hysterectomy for gynecologic oncologists without open counterpart experience. Obstet Gynecol Sci 2015; 58(5):377-84.

25. Philp L, Covens A, Vicus D, Kupets R, Pulman K, Gien LT. Feasibility and safety of same-day discharge after laparoscopic radical hysterectomy for cérvix cancer. Gynecol Oncol 2017; 147(3):572-6.

26. Park JY, Nam JH. Laparotomy conversion rate of laparoscopic radical hysterectomy for early-stage cervical cancer in a consecutive series without case selection. Ann Surg Oncol 2014; 21(9):3030-5.
27. O'Neill M, Moran PS, Teljeur C, O'Sullivan OE, O'Reilly BA, Hewitt M, et al. Robot-assisted hysterectomy compared to open and laparoscopic approaches: systematic review and meta-analysis. Arch Gynecol Obstet 2013; 287(5):907-18.

28. Laterza RM, Sievert KD, de Ridder D, Vierhout ME, Haab F, Cardozo L, et al. Bladder function after radical hysterectomy for cervical cancer. Neurourol Urodyn 2015; 34(4):309-15

29. Diver E, Hinchcliff E, Gockley A, Melamed A, Contrino L, Feldman S, et al. Minimally invasive radical hysterectomy for cervical cancer is associated with reduced morbidity and similar survival outcomes compared with laparotomy. J Minim Invasive Gynecol 2017; 24(3):402-6. 